\title{
Die Koniferen blühen - eine glitzernde Mikrowelt bei den Zypressengewächsen
}

\author{
ARMin JAGEL
}

\begin{abstract}
In contrast to most of the flowering plants (angiosperms) conifers have unspectacular flowers. Especially members of the Cypress Family have freely accessible pollination structures. Their pollination droplets are not hidden in cones. The diversity of pollination droplets of various conifers are shown.
\end{abstract}

\section{Zusammenfassung}

Koniferen blühen im Gegensatz zu den meisten Blütenpflanzen (den Bedecktsamern) unauffällig und zumeist unbemerkt. Besonders bei den Zypressengewächsen (Cupressaceae) liegen die Strukturen für das menschliche Auge frei zugänglich. Die zum Pollenfang gebildeten Bestäubungstropfen sind hier anders als bei vielen anderen Koniferen nicht tief im Zapfen versteckt. Wie genaue Beobachtungen zeigten, gibt es außerdem verschiedene Abwandlungen bei der Bildung und der Funktion der Bestäubungstropfen.

\section{Einleitung}

Die Zapfen vieler Koniferen fallen den meisten erst dann auf, wenn sie ausgewachsen sind und auf den Boden fallen. Vor allem größere Zapfen werden häufig in der Weihnachtsfloristik verwendet. Aber auch Zapfen fangen klein an. Wann eigentlich blühen Nadelbäume oder wer- den die jungen Zapfen angelegt? Im Volksmund wird manchmal derjenige Zeitraum als Blütezeit bezeichnet, bei dem die „Tannen“ im Frühjahr frischgrün austreiben. Die wirkliche Zeit der Blüte bleibt aber dem ungeübten Auge meist verborgen, da die Vorgänge versteckt im Inneren der Zapfen ablaufen. Anders verhält es

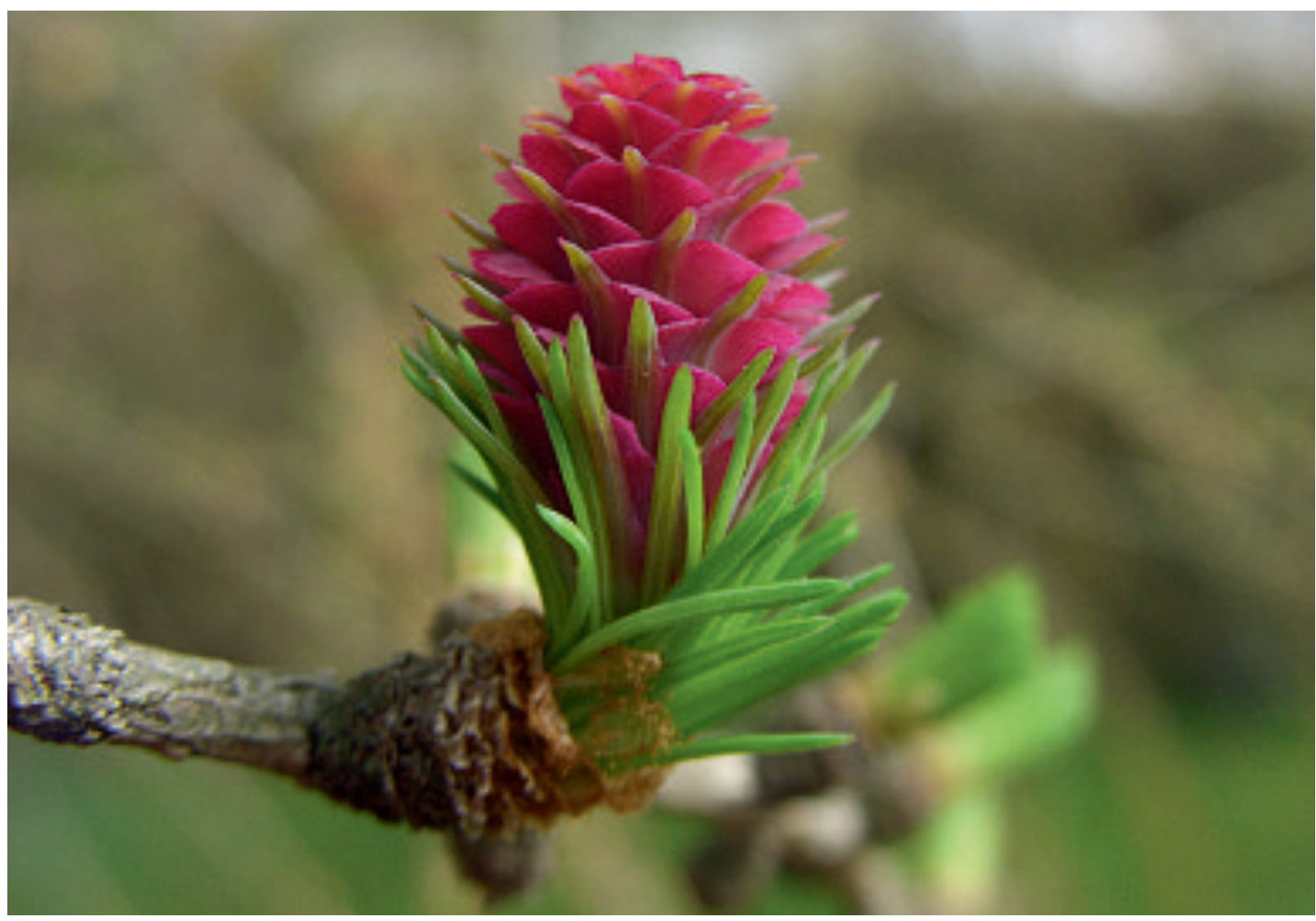




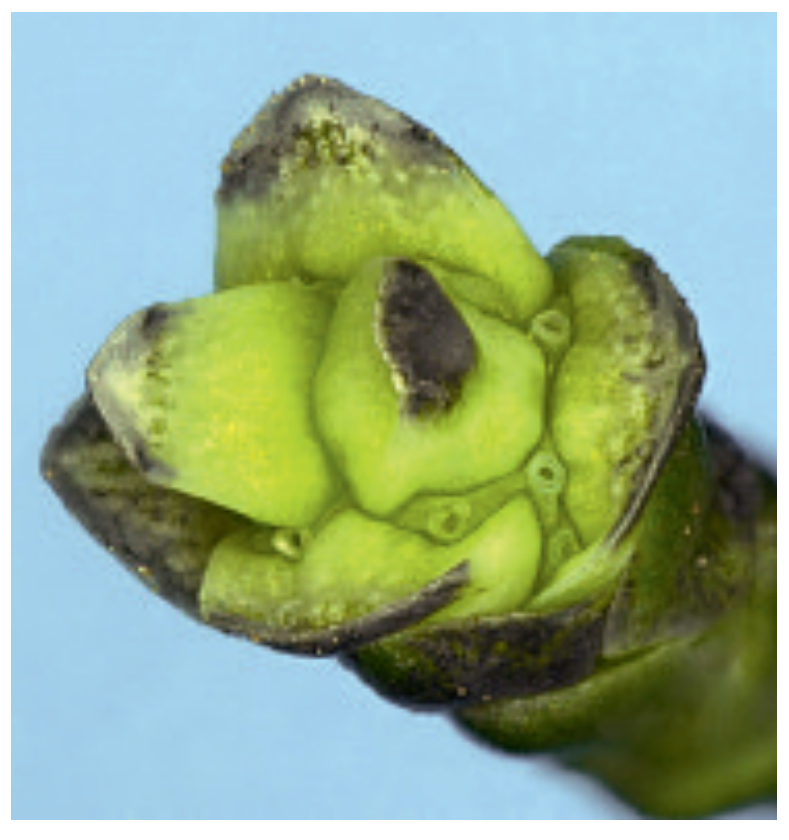

sich bei den heutigen Arten der Zypressengewächse (Cupressaceae s. str.). Hier sind die entscheidenden Vorgänge gut von außen zu erkennen, allerdings sind die beteiligten Elemente so klein, dass man oft erst einmal darauf aufmerksam gemacht werden muss. So soll im Folgenden ein kleiner Einblick insbesondere in die Mikrowelt blühender Zapfen von Zypressengewächsen gegeben werden.

\section{Blütenstände und Blüten}

Koniferen blühen in der Regel im Winter oder im zeitigen Frühjahr, bei ungewöhnlich warmem Wetter manchmal aber auch schon im Spätherbst. Die jungen Zapfen, die als Blütenstände aufgefasst werden können, sind zu diesem Zeitpunkt jedenfalls bereits vollständig angelegt.

In den meisten Fällen blühen Koniferen unauffällig. Ihre Blüten sind sparsam ausgestattet im Vergleich mit den Bedecktsamern (Angiospermen), die meist Blüten mit auffälligen Schauapparaten entwickeln wie z. B. Rose oder Mohn. Es wird oft kritisch beurteilt, wenn bei Koniferen von „Blüte“ und „blühen“" gesprochen wird, da „richtige“ Blüten erst bei den Angiospermen entwickelt werden. Es gibt bei den Koniferen weder Blütenblätter noch Fruchtknoten. Die Samenanlagen der Koniferen stehen nackt in den Achseln oder auf der Fläche einer Zapfenschuppe, weshalb sie zu den Nackt-

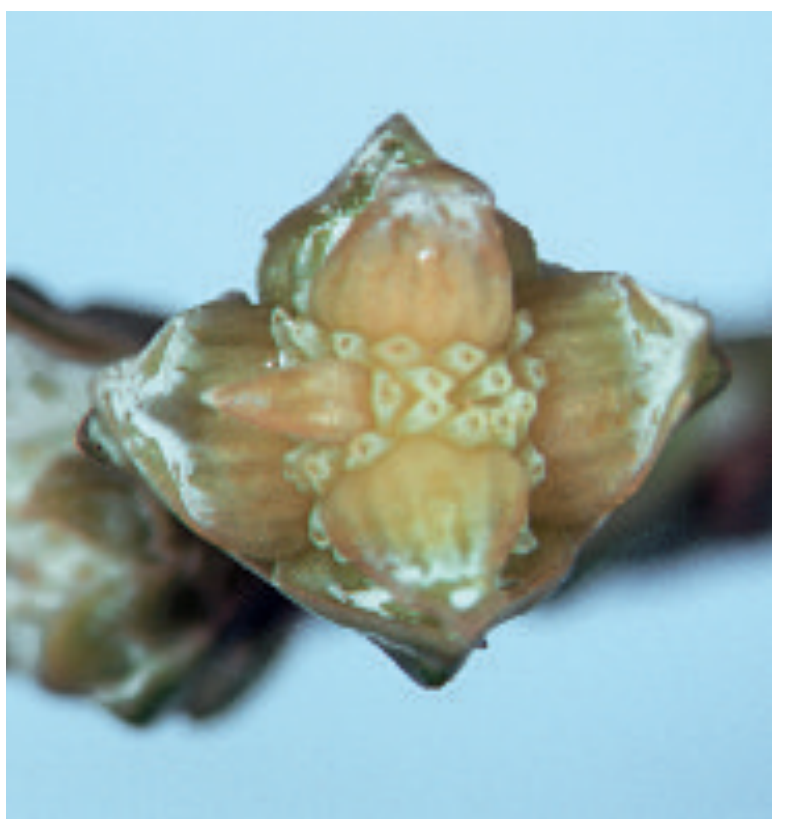

samern (Gymnospermen) gezählt werden. Es bedarf bei vielen Koniferen einiger Übung, die Blüten überhaupt zu finden. Bei den Zypressengewächsen z. B. klappen an luftfeuchten, frostfreien Tagen einfach ein paar Schuppenblätter zur Seite und geben die Samenanlagen zur Bestäubung frei (Abb. 2, 3). Zu diesem Zeitpunkt sind die Zapfen aufgrund ihrer geringen Größe kaum als solche zu erkennen. Manchmal haben die Zapfenschuppen in diesem Zustand bereits eine andere Farbe als die Blätter. Koniferen werden aber vom Wind bestäubt und müssen keine Bestäuber (z.B. durch spezielle Farbgebung) anlocken.

Auffälliger dagegen sind die meisten blühenden Zapfen der Kieferngewächse (Pinaceae), bei

Abb. 1 (Seite 137): Larix decidua (Europäische Lärche, Pinaceae): Blühender Zapfen.

Abb. 2 (links): Chamaecyparis lawsoniana (Lawsons Scheinzypresse, Cupressaceae): Zapfen zur Blütezeit. Die Zapfenschuppen spreizen ab und geben die Samenanlagen frei.

Abb. 3 (rechts): Cupressus $\mathrm{x}$ leylandii (= Cupressocyparis $\mathrm{x}$ leylandii, Leyland-Zypresse, Cupressaceae): Zapfen zur Blütezeit.

Abb. 4 (Seite 139): Riesige Mengen von Pollen windbestäubter Arten in einer Pfütze vor dem Palmengarten in der Siesmayerstraße. Die Pollenkörner haben ihr eigentliches Ziel verfehlt, nämlich die Bestäubung der weiblichen Blüten. 
denen schon aufgrund der Größe, manchmal auch an der Farbe, deutlich erkannt werden kann, dass sie blühen (Abb. 1). Die eigentlichen Blüten sind hier aber nicht zu sehen. Sie stecken tief verborgen im Zapfen, der als ein Blütenstand aufgefasst wird.

\section{Bestäubung}

Anders als bei tierbestäubten Arten ist bei Koniferen wie auch bei anderen windbestäubten Gehölzen die Bestäubung in einem hohen Maße dem Zufall überlassen. Der Pollen wird durch den Wind verdriftet, breitet sich aber ansonsten ungerichtet aus und ist völlig unabhängig von den bestäubungsfähigen Samenzapfen unterwegs. Es gibt allerdings eine Reihe von Mechanismen, die die Wahrscheinlichkeit erhöhen, dass auf der reifen Samenanlage auch wirklich ein Pollenkorn landet. Dazu wird zunächst einmal in den Pollenzapfen (männliche Blüten) eine riesige Menge an Pollen produziert (Abb. 4, 5), denn der allergrößte Teil geht un- terwegs verloren und landet eben nicht auf Samenanlagen, sondern auf dem Erdboden (Abb. 4) oder besonders augenscheinlich auch auf Fenster- oder Autoscheiben. Dies ist eine gigantische Verschwendung, wenn man so will.

Bemerkenswert an dieser Stelle ist, dass es Allergien gegen Koniferen-Pollen bei uns so gut wie nicht gibt, Allergiker können also tief durchatmen.

Die Blüten der Koniferen sind entweder einhäusig (monözisch) verteilt wie z. B. bei den Lärchen, dann existieren eingeschlechtliche weibliche und männliche Blüten auf einer Pflanze. Oder aber sie sind zweihäusig verteilt (diözisch), dann gibt es rein weibliche und rein männliche Individuen, wie das oft (aber nicht immer) bei unserem heimischen Gewöhnlichen Wacholder (Juniperus communis) der Fall ist. Zwittrige Blüten mit männlichen und weiblichen Anteilen, wie sie bei der Mehrzahl der Blütenpflanzen auftreten, gibt es bei den Koniferen nicht.

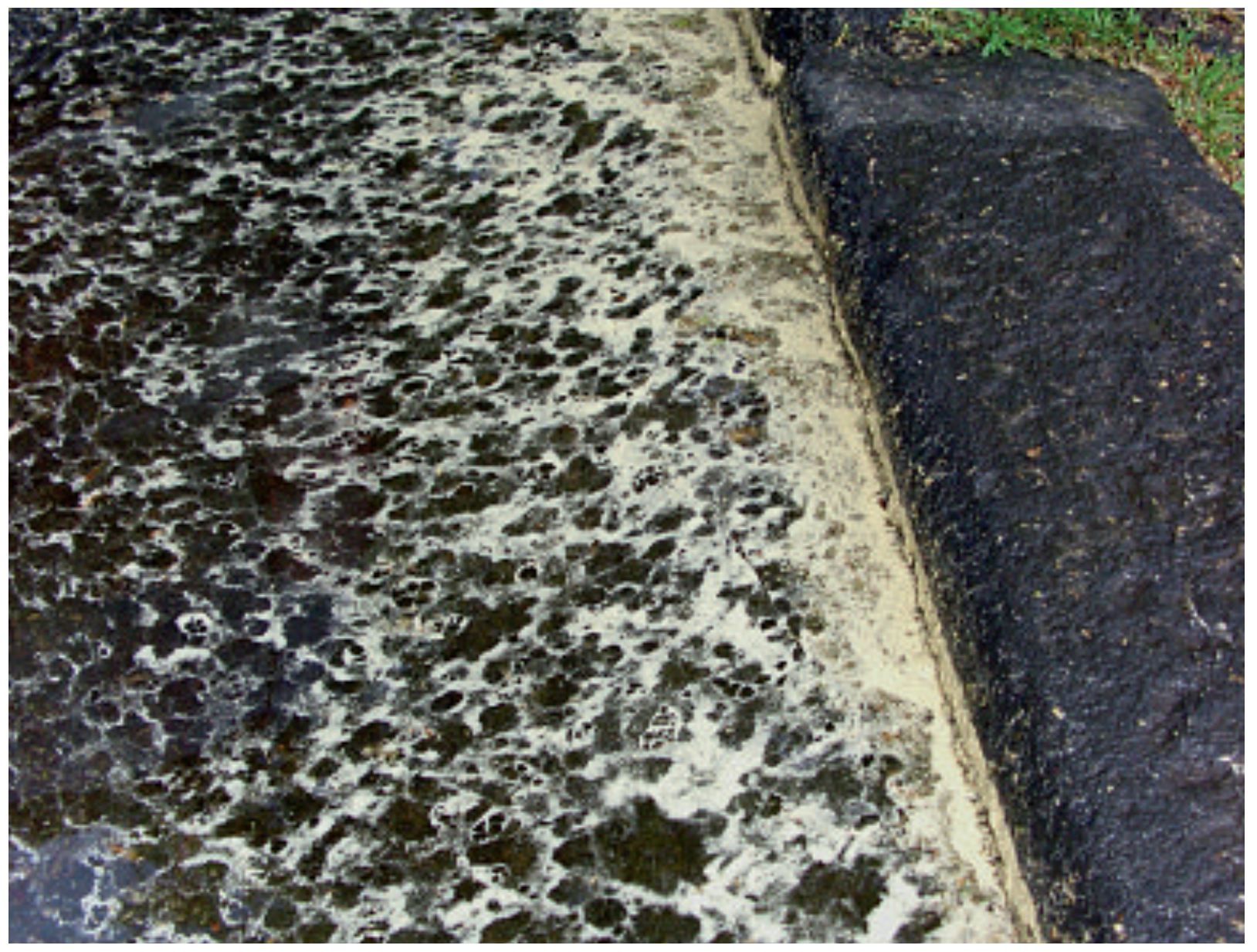




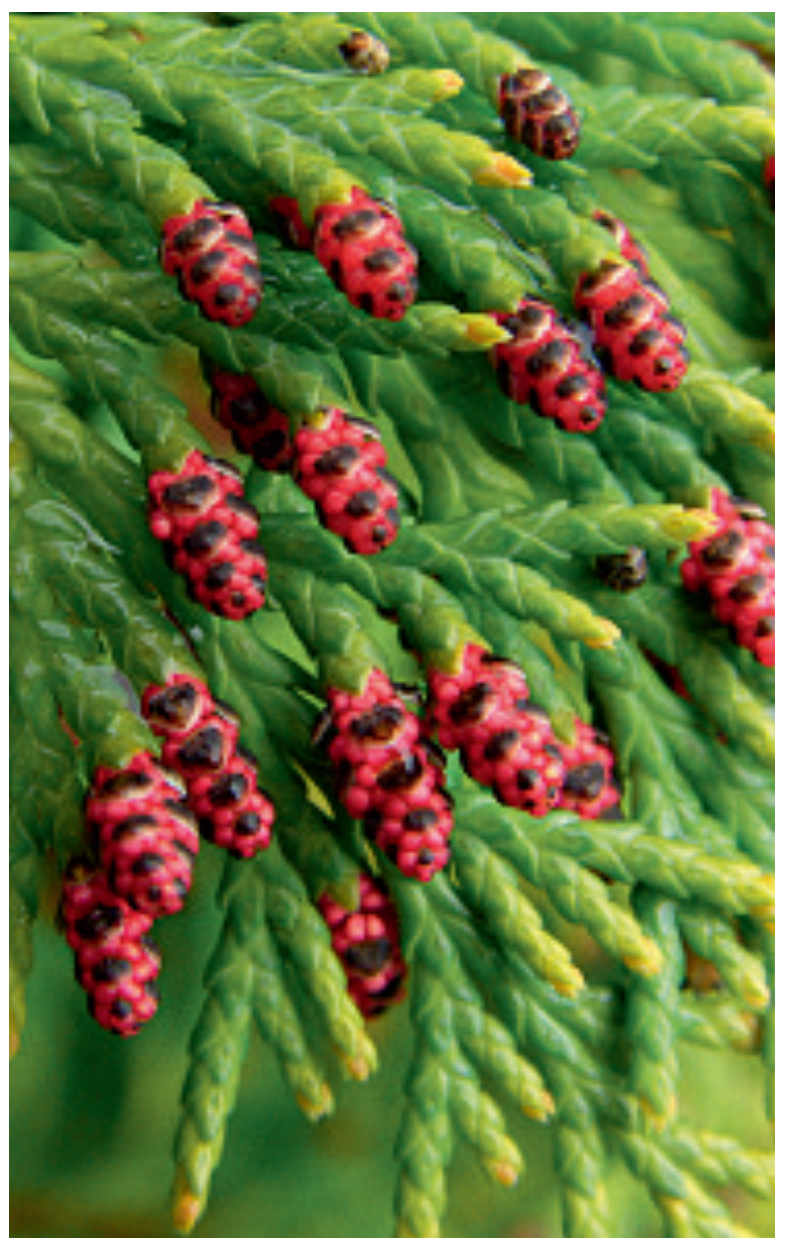

Die männlichen Blüten erscheinen durch die Farbe der Pollenkörner meist gelb. Es wird angenommen, dass Gelbtöne besonders gut UVStrahlung abhalten, deren schädigende Wirkung sich insbesondere bei Keimzellen negativ auswirken würde. Pollen kann in Einzelfällen aber auch auffälliger gefärbt sein wie z. B. bei einer unserer häufigsten Zierkoniferen, der Lawson-Scheinzypresse (Chamaecyparis lawsoniana, Abb. 5).

\section{Bestäubungstropfen}

Ein weiterer Mechanismus, um die Bestäubungswahrscheinlichkeit zu erhöhen, ist bei den Koniferen (und auch anderen Nacktsamern) entstanden, den es bei den Blütenpflanzen so nicht gibt. Es wird ein so genannter Bestäubungstropfen ausgebildet. Er entsteht in der Samenanlage und wird durch eine kleine Öffnung an der Spitze, der Mikropyle, nach außen abgesondert, um den Pollen aus der Luft zu fangen. Kurze Zeit, nachdem Pollenkörner auf dem Bestäubungstropfen aufgetroffen und in ihn aufgenommen worden sind, wird der Tropfen, zumindest bei den Cupressaceae, aktiv wieder eingezogen. Somit ist die Bestäubung vollzogen. Nun können die Pollenkörner in der Samenanlage keimen, einen Pollenschlauch ausbilden, über den die Eizelle befruchtet wird, sodass schließlich der Same heranwachsen kann.

Bestäubungstropfen sind mit bloßem Auge zu sehen, wenn man sich vergegenwärtigt, mit welcher Größe man zu rechnen hat und wo am Baum bzw. Strauch und am Zweig sie zu suchen sind. Es ist darauf zu achten, wo die reifen Zapfen des Vorjahres oder der Vorjahre stehen. Wenn man diese Position nun auf die jüngsten Zweige bezieht, sind die Chancen groß, die jüngsten Zapfenanlagen aufzuspüren. Allerdings kommt es durchaus auch vor, dass in bestimmten Jahren auf der gesamten Pflanze überhaupt keine Zapfen gebildet werden. Eine größere Chance, Bestäubungstropfen zu finden, besteht erfahrungsgemäß an einem luftfeuchten Tag und am Morgen. Die Abbildungen 6-11 vermitteln Eindrücke aus der Mikrowelt blühender

Abb. 5: Chamaecyparis lawsoniana (Lawsons Scheinzypresse, Cupressaceae): Männliche Blüten (Pollenzapfen).

Abb. 6 (Seite 141 oben links): Cupressus nootkatensis (= Chamaecyparis nootkatensis, Nutka-Zypresse): Zapfen mit Bestäubungstropfen.

Abb. 7 (Seite 141 oben rechts): Microbiota decussata (Sibirischer Zwerglebensbaum, Cupressaceae): Bestäubungstropfen. Der Zapfen enthält nur eine Samenanlage.

Abb. 8 (Seite 141 Mitte links): Thuja occidentalis (Abendländischer Lebensbaum, Cupressaceae): Zapfen mit Bestäubungstropfen.

Abb. 9 (Seite 141 Mitte rechts): Platycladus (= Thuja) orientalis (Morgenländischer Lebensbaum, Cupressaceae): Zapfen mit Bestäubungstropfen.

Abb. 10 (Seite 141 unten links): Taxodium distichum (Sumpfzypresse, Cupressaceae): Zapfen mit Bestäubungstropfen.

Abb. 11 (Seite 141 unten rechts): Juniperus chinensis (Chinesischer Wacholder, Cupressaceae): Zapfen mit Bestäubungstropfen. 

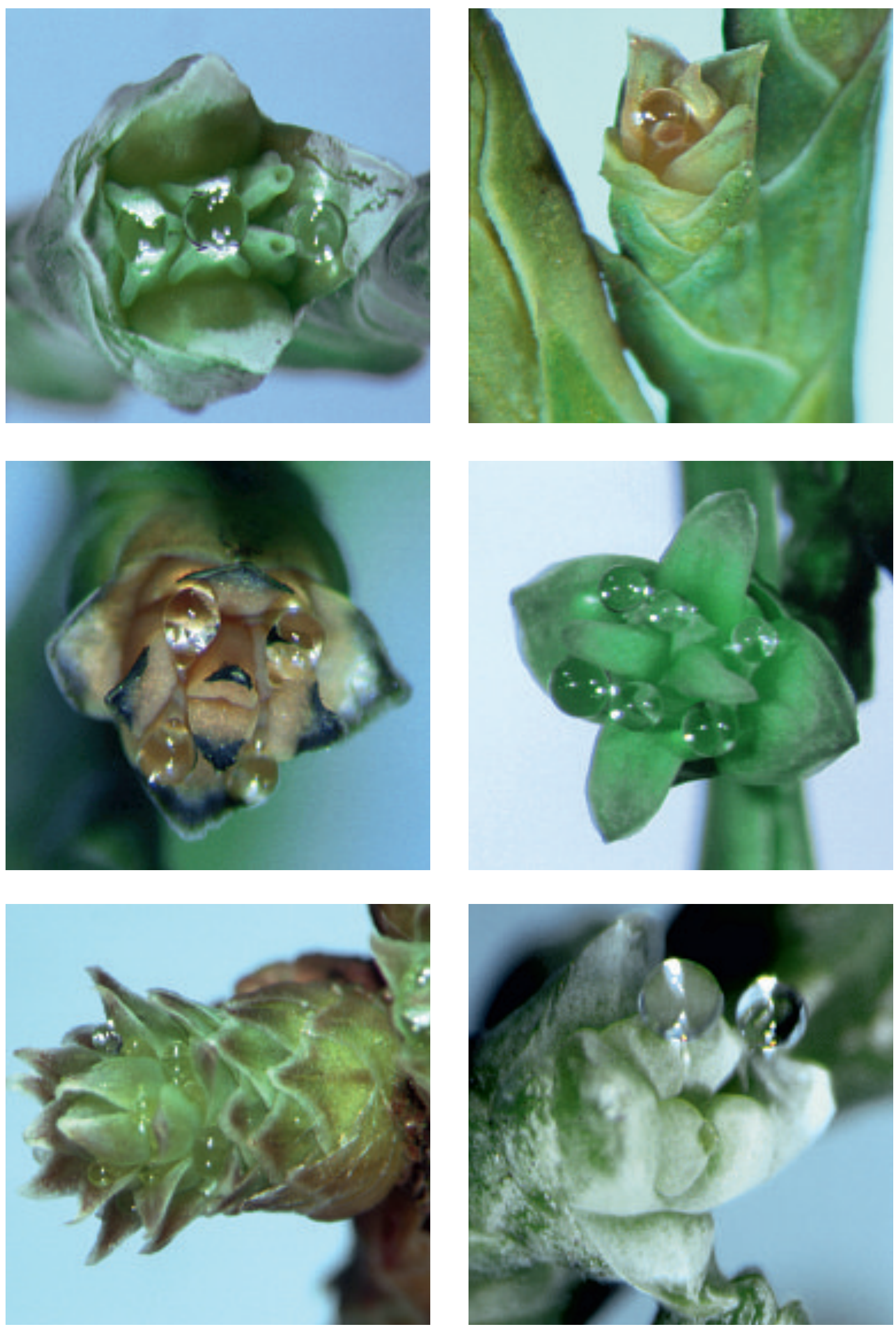

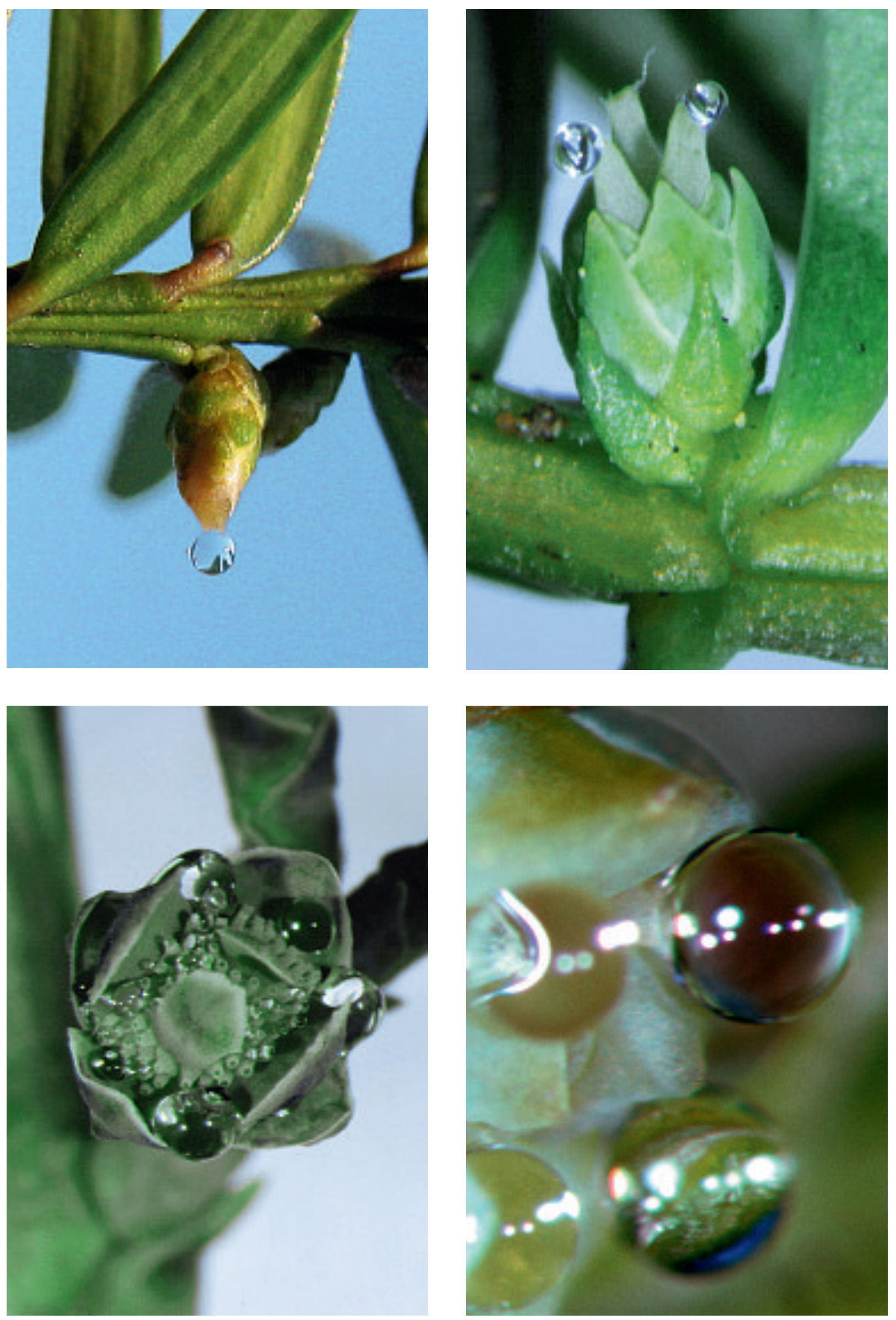


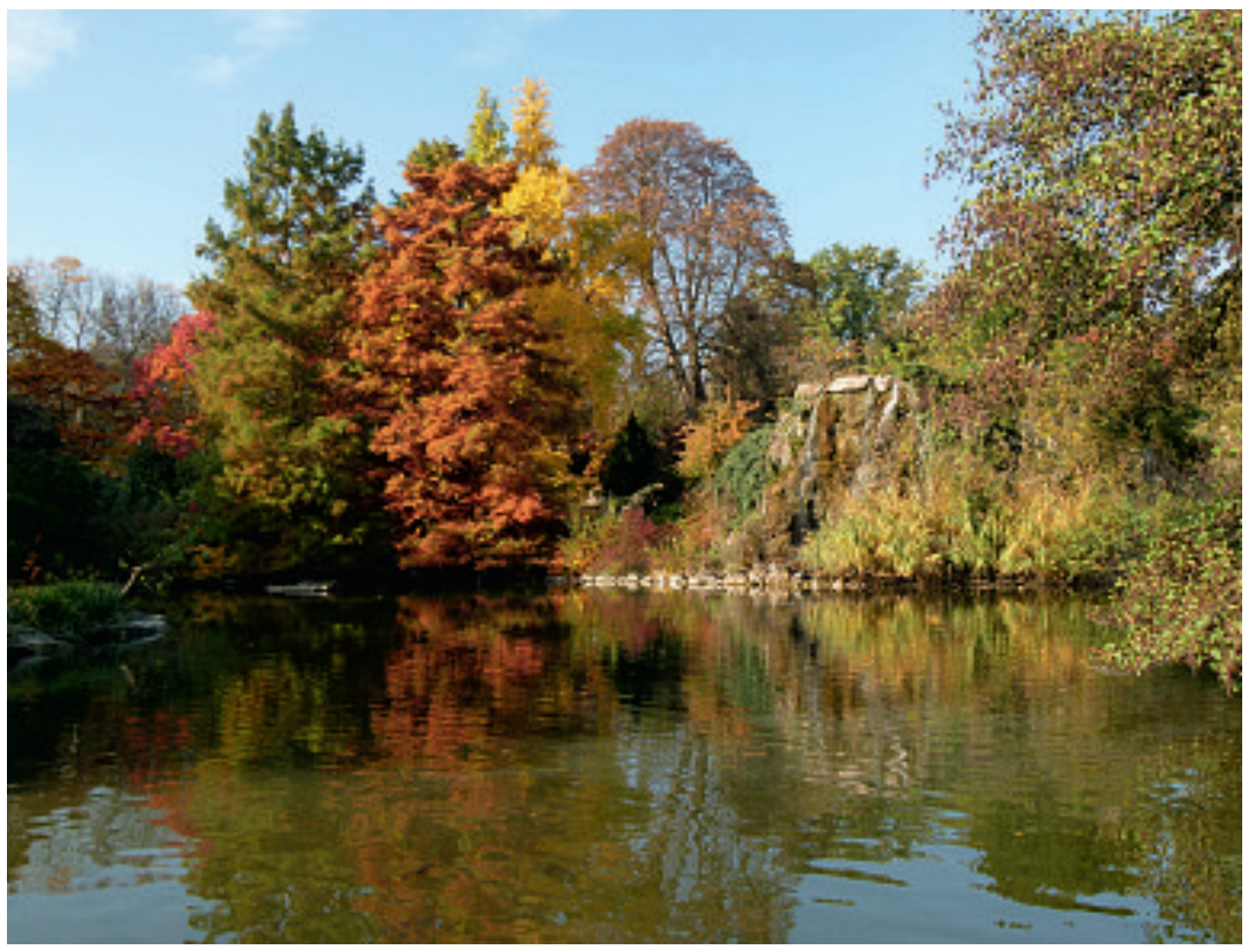

Zypressengewächse, die in unseren Gärten, Parks und auf Friedhöfen allgegenwärtig sind.

Entsprechende Bestäubungstropfen kommen (möglicherweise in einer etwas abgewandelten Funktionsweise) z. B. auch bei der Europäi-

Abb. 12 (Seite 142 oben links): Taxus baccata (Europäische Eibe, Taxaceae): Bestäubungstropfen.

Abb. 13 (Seite 142 oben rechts): Juniperus conferta (Küsten-Wacholder, Cupressaceae): Die Bestäubungstropfen werden durch eine schräge Öffnung der Samenanlage nach außen ausgerichtet, sodass die Tropfen nicht miteinander in Kontakt treten können.

Abb. 14 (Seite 142 unten links): Cupressus duclouxiana (Chinesische Zypresse, Cupressaceae) aus China: Bestäubungstropfen fließen zu größeren Tropfen zusammen.

Abb. 15 (Seite 142 unten rechts): Calocedrus formosana (Formosa-Weihrauchzeder, Cupressaceae) aus Taiwan: Die Bestäubungstropfen lehnen sich an und können dadurch größer werden.

Abb. 16: Herbstlich gefärbte Sumpfzypresse

(Taxodium distichum) am Großen Weiher im Palmengarten. schen Eibe (Taxus baccata) und ihren Verwandten (Abb. 12) sowie bei den Kiefern (Pinus) vor; bei Lärchen (Larix) und Araukarien (Araucaria) allerdings fehlen sie.

Die Zypressengewächse haben zur Steigerung der Viskosität neben der Einlagerung von Zucker weitere Mechanismen entwickelt, um die Größe des Tropfens zu erhöhen. Bei den echten Zypressen (Abb. 13) beispielsweise und bei einigen Wacholder-Arten (Juniperus) laufen die Tropfen benachbarter Samenanlagen zu einem viel größeren, gemeinsamen zusammen. Hierdurch wird die Oberfläche zum Pollenfang deutlich vergrößert. Benachbarte Samenanlagen funktionieren also quasi als gemeinsame Einheit. Dabei bekommt nicht jede beteilige Samenanlage ein Pollenkorn ab und verkümmert anschließend. Daher ist auch die Anzahl der Samenanlagen in einem Zypressenzapfen in der Regel sehr viel höher als die der letztlich herangereiften Samen. 


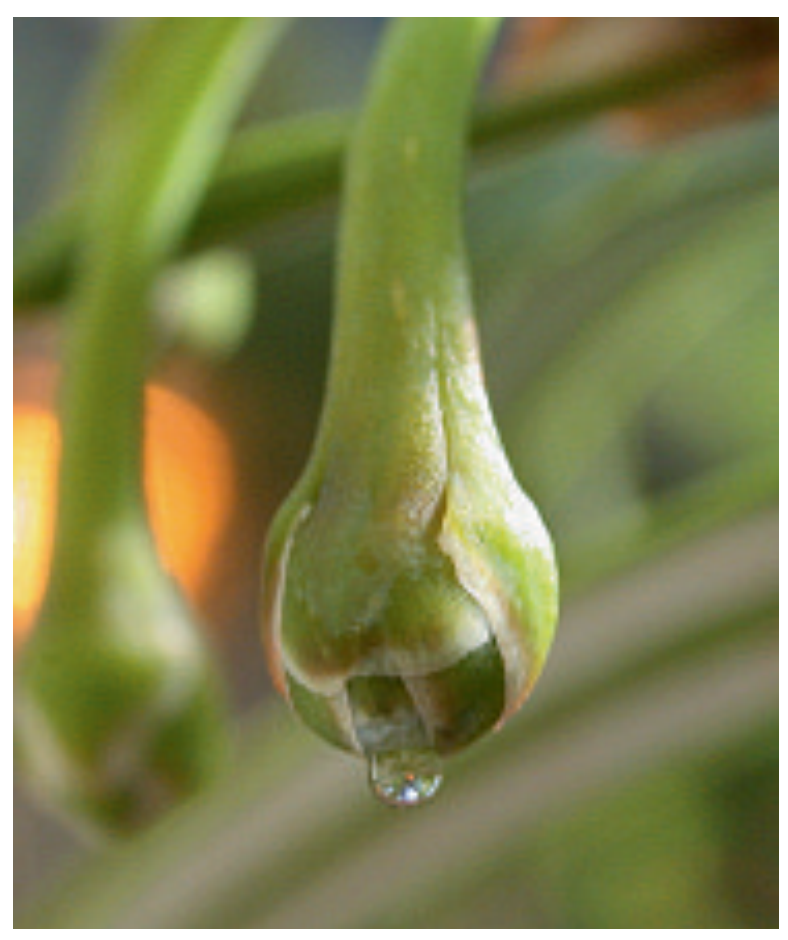

In anderen Fällen scheinen die Samenanlagen ein Zusammenfließen ihrer Tropfen zu vermeiden, wie dies z. B. bei den Wacholder-Arten der Sect. Oxycedrus der Fall ist. Hier ist die Öffnung der Samenanlage (die Mikropyle) etwas schräg ausgebildet, sodass der Tropfen nach außen zeigt und damit von den anderen Tropfen weg (Abb. 13) orientiert ist. Der normal ausgebildete Zapfen innerhalb dieser WacholderGruppe enthält nur drei Samenanlagen. Hier ist es offensichtlich sinnvoll, dass jede Samenanlage für sich alleine sorgt. Allerdings gibt es auch Zapfen mit wenigen Samenanlagen, bei denen die Tropfen zusammenfließen, wie z.B. bei der Nutka-Zypresse (Cupressus nootkatensis, vgl. Abb. 6).

Vielfach ist zu beobachten, dass Bestäubungstropfen an der unbenetzbaren, mit Wachsen überzogenen Oberfläche der Zapfenschuppe abgestützt werden. Auch hierdurch können sie erheblich größer werden (Abb. 14, 15).

\section{Schlussbetrachtung}

Viele Aspekte dieser eindrucksvollen Mikrowelt der Koniferen sind auch heute noch unbekannt oder sogar unerforscht. So dürften z. B. die Bestäubungstropfen von Amentotaxus argotaenia (Abb. 17) und von der Taiwanie (Abb.

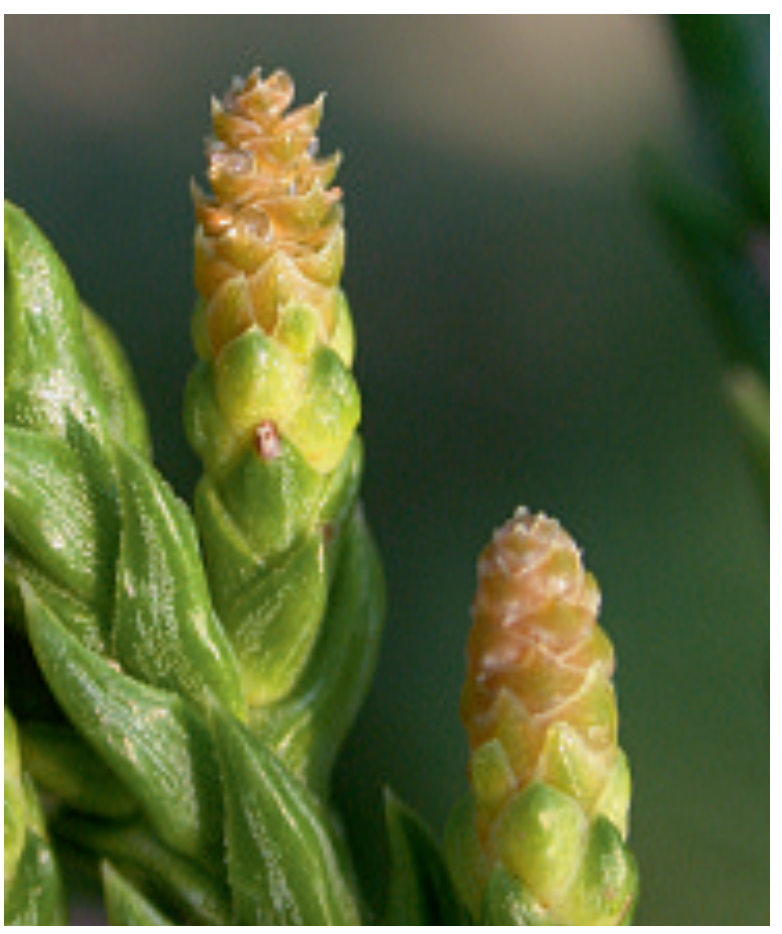

18) bisher nur selten beobachtet worden sein. Man kann sagen, dass die Koniferen sich zwar nicht mit auffälligen Blüten schmücken, dafür aber im Verborgenen unbemerkt eine kleine faszinierende Welt darbieten, mit der man sich in den Wintermonaten beschäftigen kann und die sicherlich noch einige Überraschungen bereit hält. Auch im Palmengarten ist eine artenreiche Sammlung diverser Koniferen vorhanden. Es lohnt sich auch hier, gegen Ende des Jahres nicht nur die rotorange Farbenpracht der Sumpfzypressen zu bestaunen, sondern auch in die Mikrowelt der jungen Zapfen einzutauchen.

\section{Dank}

Für die Möglichkeit, die Binokularfotos der blühenden Zapfen am Lehrstuhl Spezielle Botanik an der Ruhr-Universität Bochum anzufertigen, bedanke ich mich bei Herrn Prof. Dr. T. Stützel.

Abb. 17 (links): Amentotaxus argotaenia (Taxaceae), Bestäubungstropfen im Botanischen Garten Bonn.

Abb. 18 (rechts): Taiwania cryptomerioides (Taiwanie, Cupressaceae s.1.), Zapfen mit Bestäubungstropfen im Botanischen Garten Düsseldorf. 